\title{
Limited role of surgery in the management of primary central nervous system lymphoma (Review)
}

\author{
BO-LIN LIU ${ }^{1 *}$, JIN-XIANG CHENG $^{1 *}$, XIANG ZHANG ${ }^{1}$, WEI ZHANG ${ }^{1}$ and HONG CHENG ${ }^{2}$ \\ ${ }^{1}$ Department of Neurosurgery, Xijing Institute of Clinical Neuroscience, ${ }^{2}$ Department of Pathology, \\ Xijing Hospital, Fourth Military Medical University, Xi'an, Shaanxi Province 710032, P.R. China
}

Received March 18, 2009; Accepted June 11, 2009

DOI: $10.3892 /$ or_00000455

\begin{abstract}
Primary central nervous system lymphoma (PCNSL) is a nervous-system-seeking extranodal nonHodgkin's lymphoma whose incidence has increased in both immunocompromised and immunocompetent patients. Corticosteroids are used for symptomatic management but can interfere with pathological diagnosis. The well-established treatments such as radiotherapy, chemotherapy or a combination of both remain the mainstays. Traditionally, the most important role for surgery is to obtain and sample adequate tissue to confirm a diagnosis with stereotactic biopsy. Though no benefit could be demonstrated for debulking surgery, a subset of patients with large space occupying lesions which could be well circumscribed and surgically accessible may benefit from tumor resection. Moreover, surgical procedures to carry out interstitial chemotherapy and/or brachytherapy with or without tumor resection hold promise. Complications such as hydrocephalus could be managed by ventriculoperitoneal shunting. Further application of surgical procedures in the treatment of PCNSL is recommended with caution and strict patient selection criteria, and should be used in combination with radiotherapy and/or chemotherapy.
\end{abstract}

\section{Contents}

1. Introduction

2. Etiology and pathology

3. Diagnosis

4. Prognosis

5. Well-established treatment options

Correspondence to: Professor Xiang Zhang, Department of Neurosurgery, Xijing Institute of Clinical Neuroscience, Xijing Hospital, Fourth Military Medical University, West Changle Road, No.127, Xi'an, Shaanxi Province 710032, P.R. China

E-mail: xzhang@fmmu.edu.cn

${ }^{*}$ Contributed equally

Key words: primary central nervous system lymphoma, radiotherapy, chemotherapy, surgery, stereo-tactic biopsy, craniotomy
6. Surgical consideration

7. Conclusion

\section{Introduction}

Primary central nervous system lymphoma (PCNSL) is a rare form of non-Hodgkin's lymphoma (NHL) that is restricted entirely to the brain, leptomeninges, eyes, and rarely the spinal cord, representing $3.1 \%$ of all primary brain tumors (1). PCNSL has a higher incidence among immunocompromised patients, presenting the most common intracranial neoplasm in patients with AIDS (2). After a 3-fold rise two decades ago, the incidence of PCNSL continues to increase at a rate of 0.46 per 100,000 patient-years $(1,3)$. The increasing incidence can be partially attributed to prolonged survival of AIDS patients and the extensive use of immunosuppressive therapy in organ transplantation and autoimmune disease (4). However, there has also been a 3-fold increase in the incidence of PCNSL among immunocompetent patients, within some studies representing more than $6 \%$ of primary intracerebral neoplasms (5). This review focuses on PCNSL in the immunocompetent patient population.

Most of the current data regarding PCNSL strongly support a protocol for stereotactic biopsy to establish the most reliable diagnosis, followed by chemotherapy and radiotherapy (RT) tailored to treat the histological subtype of the tumor (6). The efficacy of chemotherapy and RT is well established but, despite a response rate up to $80 \%$, it is almost never curative with a median survival after diagnosis of 1141 months $(7,8)$. Craniotomy for debulking or resection of the tumor is widely believed to provide no therapeutic benefit $(9,10)$; however, the reasons for that are not entirely clear. Though perhaps the most salient argument against aggressive resection is the microscopically widespread nature of PCNSL at diagnosis, there is a possibility that the tumors respond so well to chemotherapy and RT, that the benefits of surgery may be underestimated. Although the tumors may disappear after chemotherapy and RT, they usually relapse, so that a closer look to the role of surgery is warranted.

In this reviews, in addition to chemotherapy, RT and stemcell rescue, we paid more attention to the questions concerning the surgical management of PCNSL, such as patient selection, preoperative workup, the diagnostic value of stereotactic biopsy, and the therapeutic role of open craniotomy. 
Table I. Clinical signs and different lesion sites at admission in patients with PCNSL.

\begin{tabular}{lc}
\hline Clinical signs & $(\%)$ of patients \\
\hline Focal neurologic deficits & 70 \\
Neuropsychiatric symptoms & 43 \\
Increased intracranial pressure & 33 \\
Seizures & 14 \\
Ocular symptoms & 4 \\
Leptomeningeal involvement & $16-41$ \\
Lesion sites & \\
Hemispheres & 38 \\
Thalamus/basal ganglia & 16 \\
Corpus callosum & 14 \\
Ventricular region & 12 \\
Cerebellum & 9
\end{tabular}

\section{Etiology and pathology}

PCNSL impacts two distinct populations. Here we discuss the immunocompetence with unknown etiology. Some have postulated that PCNSL occurs secondary to a systemic malignancy that is eliminated by the peripheral immune system except for the CNS because of the immune privilege (11). Alternatively, it has been proposed that a malignant clone of systemic lymphocytes expressing specific adhesion molecules may enter the brain $(12,13)$.

PCNSL makes up $<2-3 \%$ of NHLs (14), whilst being classified as stage IE NHL because of the restriction to a single extranodal site. The most common histopathological diagnosis is malignant diffuse large B-cell lymphomas (DLBCLs) which are germinal center in origin, accounting for $90 \%$ of all PCNSL (15). T-cell variants are rare and account for $<4 \%$ of all PCNSL in the Western world (11), whose clinical presentation and outcome appear very similar to that of B-cell PCNSL (16). Another even rarer form primary low grade CNS lymphoma has been reported in the literature as small series or single case reports, which represent classically as dural-based masses mimicking meningioma and seem to have a better prognosis (17).

\section{Diagnosis}

Clinical presentation. The clinical presentation of patients with PCNSL varies according to the location and size of the tumor, as well as the immunological status of the patient. In immunocompetent patients with PCNSL, the median age at presentation is 55 years, and the male-to-female ratio has been reported as ranging from 1.2:1 to 1.7:1 (9).

Similar to brain tumors in general, patients with PCNSL, of whom $65 \%$ were solitary lesions and $35 \%$ were multifocal lesions, typically present with focal symptoms and raised intracranial pressure (Table I) $(6,9)$.

Patients with intraocular lymphoma (IOL) may complain of blurry vision, clouded vision or floaters. If lymphoma is not considered during slit-lamp examination, these patients have a large possibility to be misdiagnosed with uveitis. Patients with leptomeningeal lymphoma are mostly asymptomatic. A specific history of truncal anaesthesia, bladder control problems, or non-localized pain should be obtained to assess signs and symptoms of invasion.

Neuroimaging. Since the presentation of PCNSL is nonspecific, a gadolinium-enhanced magnetic resonance imaging (MRI) of the brain is required for clinical decision making. PCNSL is usually hypointense to isointense on T1 images and isointense to hyperintense on T2 images. Contrast enhancement is diffuse and, because of their indistinct borders, these tumors are often appeared cloud-like (Fig. 1). Gadolinium-enhanced MRI of the spine should be done in patients with spinal cord or leptomeningeal symptoms but is not required for routine workup. Magnetic resonance spectroscopy identifies tumor masses with marked increase in choline to creatine ratio, reduction of $\mathrm{N}$-acetylaspartate and presence of a lactate-lipid peak. PET and SPECT imaging might be useful tools in screening occult systemic disease.

CSF. CSF analysis including cell counts, protein and glucose levels, cytology, flow cytometry, and immunoglobulin heavychain gene rearrangement studies, if not done for diagnosis, is necessary to evaluate for evidence of leptomeningeal involvement. Tumor diagnostic markers such as lactate dehydrogenase, $\beta$-glucuronidase, and $\beta 2$-microglobulin can be helpful (11). The detection of lymphoma cells in the CSF or in a vitreous biopsy when possible is sufficient to make the diagnosis without the need for a brain stereotactic biopsy (18). However, the initial CSF cytologic studies were positive in only two thirds of patients, suggesting a need for serial lumbar punctures; patients with rapidly worsening clinical symptoms should undergo stereotactic biopsy immediately for definitive diagnosis.

Systemic examination. Since PCNSL may involve any part of the craniospinal axis including the eyes, spinal cord, leptomeninges and CSF, all areas must be evaluated for the presence of tumor prior to making a therapeutic choice. Systemic involvement is so rare at onset $(<5 \%)$ that the need for extensive initial staging has been debated; however, Abrey et al suggested that the diagnostic workup recommended by International PCNSL Collaborative Group be followed (Table II) (19). Moreover, systemic disease should be ruled out prior to cranial biopsy, since the finding of extracranial lesion can obviate the need for a cranial procedure.

\section{Prognosis}

The identification and evaluation of prognostic factors enables stratifying PCNSL patients into specific risk groups that can help to define optimal therapy strategy and predict survival. In addition, the use of prognostic factors is critical for comparing clinical trials results which are hardly ever randomized, because of the low incidence of PCNSL.

Age, performance status, serum lactate dehydrogenase (LDH) level, CSF protein concentration and tumor location are thought to be prognostic factors for PCNSL. Lower age 

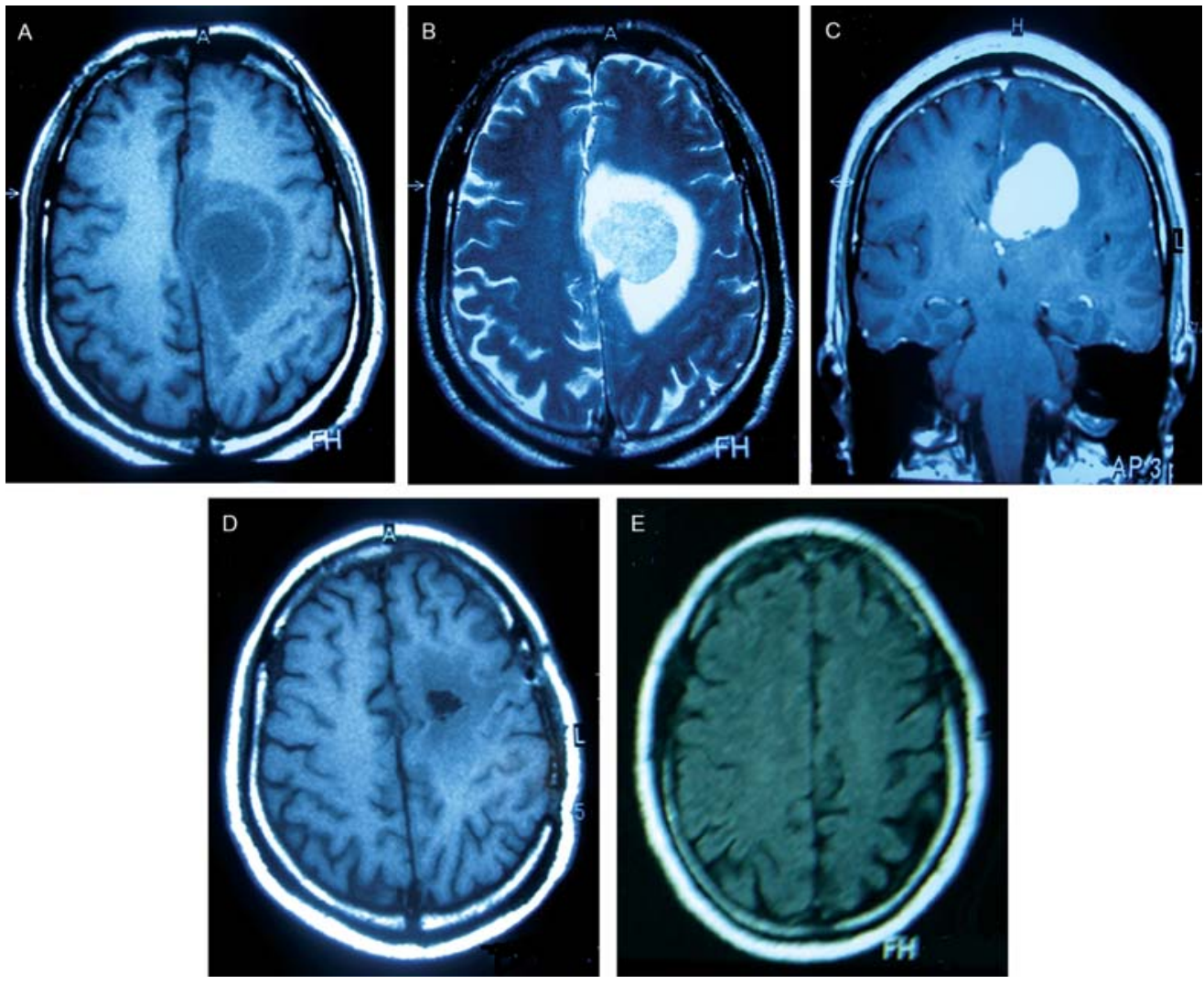

Figure 1. Contrast-enhanced MR images. A 40-year-old man was admitted with a 10-day history of headache, nausea and vomiting. A nervous system examination revealed clear consciousness; increased memory loss; normal cranial nerve functions; decreased myodynamia, hyperreflexia of right body, and a positive right Babinski's sign. Axial (A and B) and coronal (C) images showing a large enhancing mass in the deep site of the left frontal-parietal lobe (adjacent to midline). Surrounding edema and mild midline shift are also evident. The lesion is hypointense on T1-weighted images (A) and hyperintense on T2-weighted images (B). Given the signs of increased intracranial pressure and acute neurological deterioration caused by the space-occupying lesions, an instant craniotomy for tumor resection was performed. Postoperative axial image (D) revealing the solitary tumor (4x5x6 $\mathrm{cm}$ in volume) was totally removed Histopathological examination confirmed the diagnosis of DLBCL. The patient received WBRT 2 weeks postoperative, and then chemotherapy infusion via the internal carotid artery 6 weeks postoperative. Axial image (E) obtained in a follow-up survey up to 8 years found no relapse.

Table II. Recommended tests for staging and prognostication of PCNSL.

\begin{tabular}{|c|c|c|c|c|}
\hline & Pathology & Clinical & Laboratory & Imaging \\
\hline \multirow[t]{3}{*}{$\begin{array}{l}\text { CNS } \\
\text { staging }\end{array}$} & $\begin{array}{l}\text { Histological and } \\
\text { immunohistochemical } \\
\text { analysis }\end{array}$ & Neurologic examination & $\begin{array}{l}\text { CSF protein and cytology, } \\
\text { flow cytometry, immuno- } \\
\text { globulin heavy chain gene } \\
\text { rearrangement analysis }\end{array}$ & $\begin{array}{l}\text { Gadolinium-enhanced } \\
\text { cranial MRI }\end{array}$ \\
\hline & & $\begin{array}{l}\text { Opthalmologic evaluation } \\
\text { with slit lamp examination }\end{array}$ & & $\begin{array}{l}\text { Gadolinium-enhanced } \\
\text { spinal MRI (if clinically } \\
\text { indicated) }\end{array}$ \\
\hline & & $\begin{array}{l}\text { Serial evaluation of } \\
\text { cognitive function }\end{array}$ & & \\
\hline \multirow[t]{4}{*}{$\begin{array}{l}\text { Systemic } \\
\text { staging }\end{array}$} & & $\begin{array}{l}\text { General physical examin- } \\
\text { ation including evaluation } \\
\text { of lymph nodes and testicles }\end{array}$ & HIV testing & $\begin{array}{l}\text { Computed tomography of } \\
\text { chest, abdomen and pelvis }\end{array}$ \\
\hline & & $\begin{array}{l}\text { Record age and performance } \\
\text { status }\end{array}$ & $\begin{array}{l}\text { Serum lactate } \\
\text { dehydrogenase }\end{array}$ & $\begin{array}{l}\text { Bone marrow biopsy } \\
\text { with aspirate }\end{array}$ \\
\hline & & $\begin{array}{l}\text { Record corticosteroid } \\
\text { dosing }\end{array}$ & $\begin{array}{l}\text { Evaluation of hepatic } \\
\text { and renal function } \\
\text { (in patients who will } \\
\text { receive HD-MTX) }\end{array}$ & $\begin{array}{l}\text { Testicular ultrasound } \\
\text { (in older men) }\end{array}$ \\
\hline & & & & $\begin{array}{l}\text { 18F-fluorodeoxyglucose } \\
\text { PET (alternatively) }\end{array}$ \\
\hline
\end{tabular}




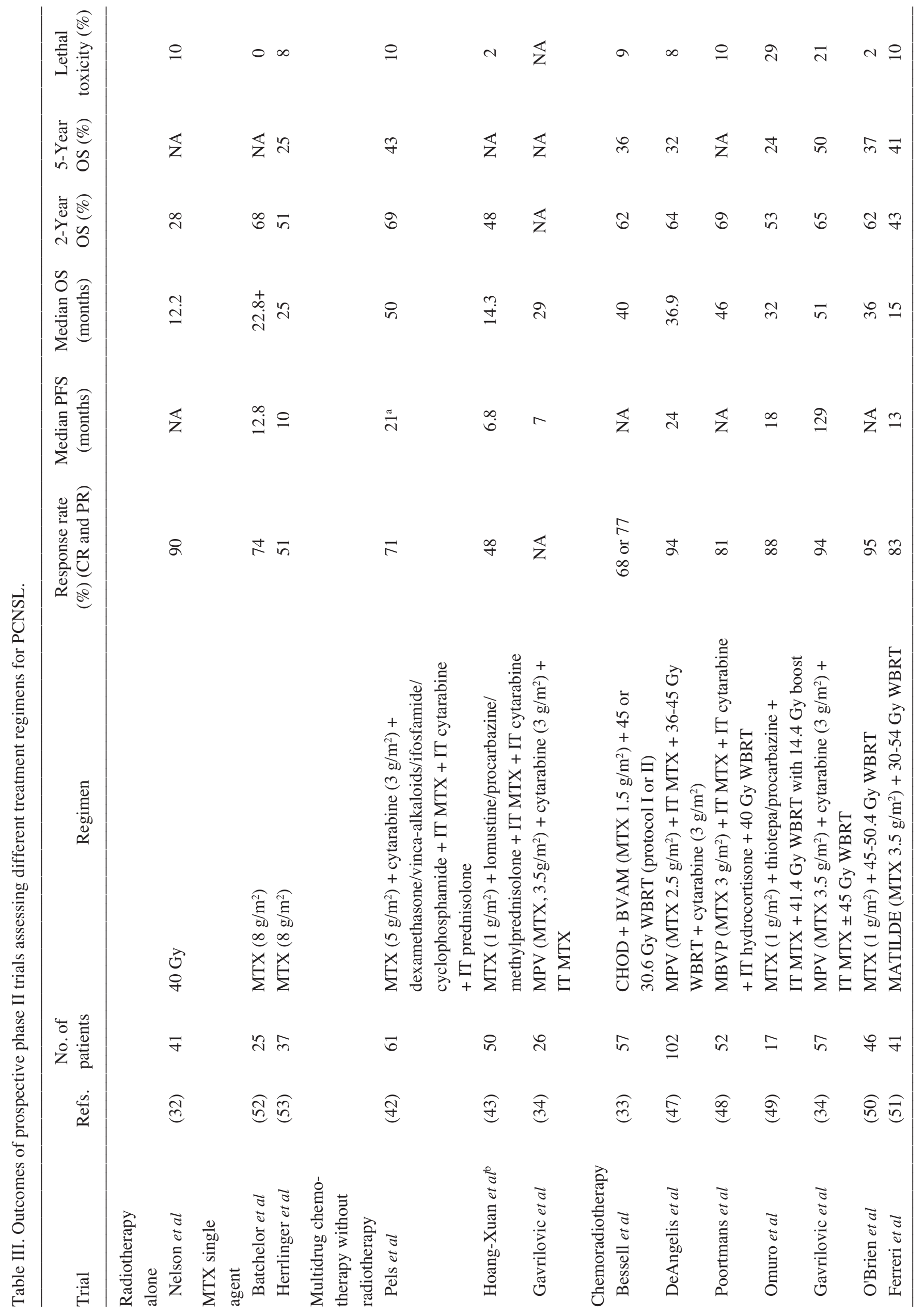




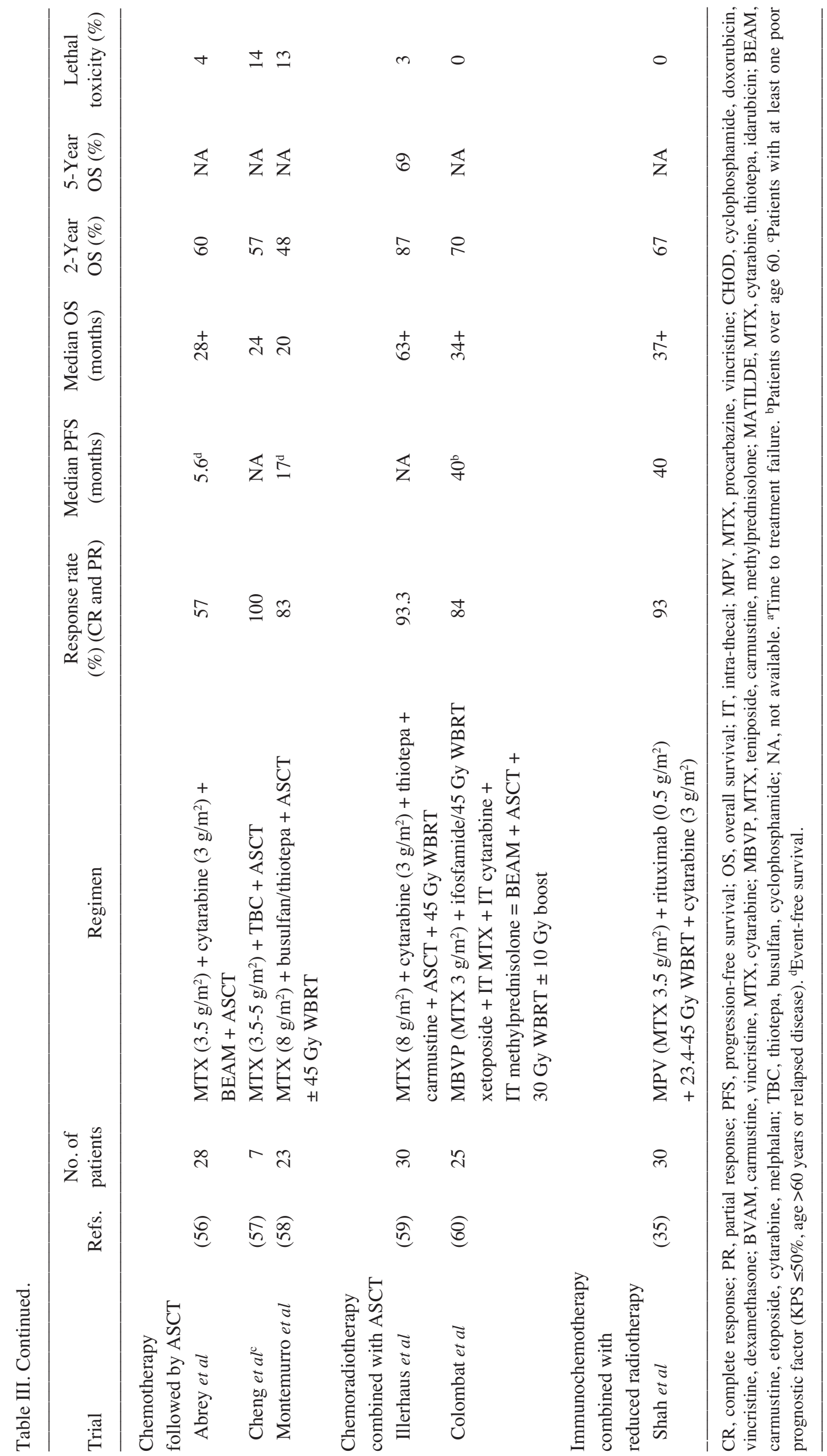


and higher Karnofsky Performance Status (KPS) have long been identified as significant favorable prognostic factors for PCNSL (20). Some even have argued that age may influence the outcome more than treatment (21). High LDH level has been a common indicator of aggressiveness and poor prognosis for all subtypes of NHL (22). Factors specific to PCNSL include elevation of CSF protein which has been shown to correlate strongly with poorer survival (22). Location of tumor in deeper structures of the brain predicts worse outcomes (22).

The International Extranodal Lymphoma Study Group (IELSG) prognostic score consists of the 5 parameters mentioned above, according to which patients are divided into 3 risk groups (23). Patients in the lowest risk group, with 1 or less unfavorable prognostic factors, have a 2-year survival of $80 \pm 8 \%$, those with 2 or 3 prognostic factors have a 2 -year survival of $48 \pm 7 \%$, and those with 4 or 5 prognostic factors have a 2 -year survival of $15 \pm 7 \%$. A model based only on age and KPS score that was developed at the Memorial SloanKettering Cancer Center divides patients into 3 groups: class 1 (age $<50$ ), class 2 (age $\geq 50$ and KPS $\geq 70$ ), and class 3 (age $\geq 50$ and KPS <70) (24). It predicts a median survival of 8.5 , 3.2 and 1.1 years for classes 1,2 and 3, respectively. Authors of this model argued that, unlike the IELSG score, their approach is simple to use and is validated by using data from prospective trials (24). Nonetheless, according to the analysis of Ferreri et al (25) the simplicity of this approach could be to the detriment of accuracy (25).

\section{Well-established treatment options}

Steroid therapy. Corticosteroids are highly effectual for symptomatic management of PCNSL. Clinical improvement after initiation of corticosteroids therapy is chiefly due to decreased edema in the surrounding brain tissue and partially due to the lympholytic effects of this compound. Corticosteroids may even cause complete resolution of lesions within a few days after initiation of treatment (26). Unfortunately, most patients relapse quickly when corticosteroids are used as the sole therapy.

It is commonly emphasized that patients with suspected PCNSL should not receive corticosteroid previous to tissue diagnosis because the lympholytic effects of these medications may lead to a non-diagnostic biopsy $(26,27)$. The histopathological appearance may be interpreted as inflammatory changes other than malignancy because of the rapid killing of B cells. Steroid treatment may also result in the findings of demyelination and the presence of $\mathrm{T}$ cells within the specimen, producing a false negative result. However, recently Porter et al (28) challenged this premise that the majority of PCNSL patients who received previous corticosteroids did not experience higher non-diagnostic biopsy rates. Thus when symptoms are severe or life-threatening (from increased intracranial pressure), steroids are the main treatment for symptomatic relief and to prevent herniation. The common strategy of the steroid treatment is to maintain patients on the minimum dose for a shortest period as possible in an effort to avoid high-dose and long-term side effects.

Radiotherapy. Neuroimaging would suggest that some of PCNSL represent focal leisions and could therefore be addressed with local therapy. Autopsy studies have confirmed that MRI significantly underestimates the extent of involvement and that PCNSL in fact is a diffuse infiltrative condition (29). Patients treated with focal RT had significant rates of relapse outside of the RT field, and even in treated ports (30). Whole-brain radiation therapy (WBRT) has consequently long been a standard component of treatment, yet the dose has been controversial. Whether RT is placed as exclusive treatment or consolidation after chemotherapy, the ideal dose based on several studies is somewhere in the range of 40-50 Gy $(20,31,32)$. Patients receiving < 40 Gy had poorer survival outcomes due to inefficient tumor control, and those receiving $>50$ Gy had poorer outcomes caused by neurotoxicity $(20,31,33)$. No benefit was shown by the addition of a boost to the tumor bed (32). Current recommendations are to treat the whole brain with 45 Gy with no boost (11). WBRT alone does not produce durable remissions for most patients (32). In patients over age 60, RT may be deferred with the aim of limiting neurotoxicity without compromising survival (34). Recent protocols have further examined the option of either deferring RT or using a lower dose in the elderly and in patients with a complete response (CR) to chemotherapy $(33,35)$.

Chemotherapy alone. The use of cyclophosphamide, doxorubicin, vincristine, and prednisone showed no benefit over RT alone, and responses tended to be transitory (36). Similar results were found when low-dose methotrexate (MTX) and bleomycin were added to the regimen (37). When used in high doses $\left(1-8 \mathrm{~g} / \mathrm{m}^{2}\right)$, MTX is essentially forced across the blood brain barrier to reach effective levels in the brain and in CSF. There is a general agreement that HD-MTX with folinic acid rescue is the most active drug available currently for PCNSL (38), while HD-MTX (>3.5 g/ $\mathrm{m}^{2}$ ) is thought to be an essential component of any treatment regimen (39). Besides, high-dose cytarabine might be an independent positive prognostic factor for improved survival if given with HD-MTX $\left(>3 \mathrm{~g} / \mathrm{m}^{2}\right.$ ) (preferably after MTX rather than before) $(40,41)$.

Chemotherapy alone recently has been emphasized again, especially in PCNSL patients older than 60 who were at highest risk for RT-related neurotoxicity. Some methotrexatebased, multiagent chemotherapy regimens have been assessed in prospective phase II trials, in which promising results were gained (Table III) $(42,43)$.

The addition of intrathecal chemotherapy makes no improvement in outcome of patients who receive HD-MTXbased chemotherapy $(38,44,45)$, while it may be worse for increased risks of neurotoxicity and chemical meningitis. Currently, intrathecal chemotherapy is added only to the regimen of patients who have a positive CSF cytologic examination.

Though chemotherapy alone is an effective treatment for some PCNSL, Bessell et al (18) contended that chemotherapy alone should be the subject of experimental protocols and not considered a standard approach in patients with IOL.

Combined-modality therapy. HD-MTX-based combination chemotherapy together with RT and treatment of leptomeningeal disease noticeably prolongs survival when 
compared with RT alone (46-51). Although most additional drugs have no proven benefit over HD-MTX alone $(15,40)$, HD-MTX alone without immediate RT afterwards appears to produce an inferior result $(52,53)$. Any regimen without HDMTX performs no better than RT alone $(36,54)$, while RT alone is insufficient to produce durable remissions for most patients (32) or to treat disease involving the subarachnoid space (46). Accordingly, it is advocated that followed by WBRT, HD-MTX alone or combinations including this drug should be used as primary approaches against PCNSL (15).

Current efforts focus on optimizing chemotherapy with the goal of deferring/reducing RT and its consequent neurotoxicity. The application of high-dose chemotherapy followed by autologous stem-cell transplantation (ASCT) is such an approach, which has succeeded in the treatment of systemic NHL (55) and holds much promise for patients with PCNSL (56-58). Alternatively, excellent disease control could be achieved by immunochemotherapy with reduceddose WBRT (35). The use of chemotherapy and ASCT in conjunction with WBRT has also resulted in prolonged survival $(59,60)$.

Salvage therapy. At relapse, some PCNSL remain treatmentsensitive to previous therapy. In patients who experienced a prolonged lymphoma remission after first-line chemotherapy containing HD-MTX, a second course may reinduce a response in over half of patients (61). However, retreatment may raise the risk for neurotoxicity. Salvage monochemotherapy with temozolomide or topotecan in patients previously managed with an RT-containing approach is supported by prospective trials, whereas the combination chemotherapy remains investigational (62-64). High-dose chemotherapy supported by ASCT and the use of intrathecal chemotherapy in patients with meningeal failure hold promise to be further addressed in prospective trials (64).

WBRT as a salvage therapy after failure with initial MTXbased chemotherapy can be correlated with a substantial rate of response and a modest neurotoxicity rate if total RT dose is <36 Gy (65). However, it should be noted that in these patients, the risk of neurotoxicity after salvage RT (in 15$58 \%$ of cases) $(53,65)$ could be greater than with RT given immediately after chemotherapy (in $8-13 \%$ of cases) $(45,66)$.

Treatment-related neurotoxicity. Despite the successful response to PCNSL management, it is not without consequences. Most important is the delayed neurotoxicity seen as a consequence of the WBRT-containing regimens (67), since chemotherapy only regimens seem to achieve lower neurotoxicity rates. However, this comparison appears biased in favor of the latter, because toxicity data here refer only to patients with $\mathrm{CR}$ after chemotherapy, while ignoring partial responders or non-responders to chemotherapy who subsequently have CR with WBRT in chemoradiotherapy regimens (68).

Symptoms can emerge as early as weeks to months after treatment, which is more prominent in the elderly and can be devastating. Clinical features comprise executive dysfunction, behavioral changes, ataxia and urinary incontinence which is progressive, while MRI shows a significant leukoencephalopathy (69).
Noticeably, the neurocognitive risk is increased in more than an additive manner when treatments are combined (70). Combination chemotherapy elevated the risk of treatment related deaths respecting single agent HD MTX from 0-3\% $(31,45,66)$ to $6-16 \%(33,42,43,48)$.

A constant dilemma in PCNSL management is the option between dose-intensified strategies to improve outcome versus deescalated approaches to avoid severe neurotoxicity. The latter continues as an increasingly significant objective when making the treatment options and is especially imperative in encouraging research for the individualization of the pathophysiological mechanisms of neurotoxicity and for risk factors pointing to neuroprotective or rehabilitative therapies aimed to prevent or treat cognitive deficits. Baseline neuropsychological tests should be carried out before treatment and repeated during and after treatment.

\section{Surgical consideration}

Stereotactic biopsy. Surgical intervention pivots on a suspected diagnosis of PCNSL, based on acute neurological presentation and neuroimaging findings. Unless there is an urgent need for surgical decompression, a biopsy to confirm a diagnosis of PCNSL is the first step in surgical management.

Biopsies of intracranial PCNSLs can be performed via frame-based or frameless stereotactic protocols, depending primarily on the surgeon's experience and the availability of necessary apparatus. In accordance with Elder and Chen (71), frame-based biopsies are preferably performed in cases involving small $(<2 \mathrm{~cm})$, deep midline lesions and in tumors that enhance well with contrast. Frameless systems are often used for a tumor in the temporal lobe (where it is more difficult to achieve a good trajectory with a frame-based system) and for larger lesions (>2 cm) (71).

The biopsy samples obtained during the procedure are sent for pathological analysis. Frozen section of the lesion with standard hematoxylin and eosin staining is of diagnostic value in those PCNSL strongly suspectable cases. Histological and immunohistochemical analysis of paraffin-embedded tissue is also performed using the following stains and antibodies: hematoxylin and eosin (Fig. 2A), glial fibrillary acidic protein (a marker for glial neoplasms), CD3 (a T-cell marker), CD20 (a B-cell marker) (Fig. 2B), CD45 (a common leukocyte antigen, which stains both $\mathrm{T}$ and $\mathrm{B}$ cells) (Fig. 2C), keratin (a marker for carcinoma), and Ki-67 (a proliferation marker) (Fig. 2D). These analyses solidify the diagnosis of PCNSL and may further categorize the tumor founded on immunological cell surface markers. It is of great significance when designing future therapeutic approaches specific to tumor subsets.

Craniotomy. Stereotactic biopsy is considered by most authors to be the only surgical intervention indicated for patients with PCNSL. Resection is usually not considered due to the multicentric, infiltrative nature of the tumor and the usual remarkable response to chemotherapy and RT. Gross-total resection or debulking of PCNSL is widely believed to offer no additional clinical or survival benefit over biopsy alone $(6,10,19)$. Murray et al $(72)$ reported a series of 11 cases of PCNSL and reviewed the previously reported 

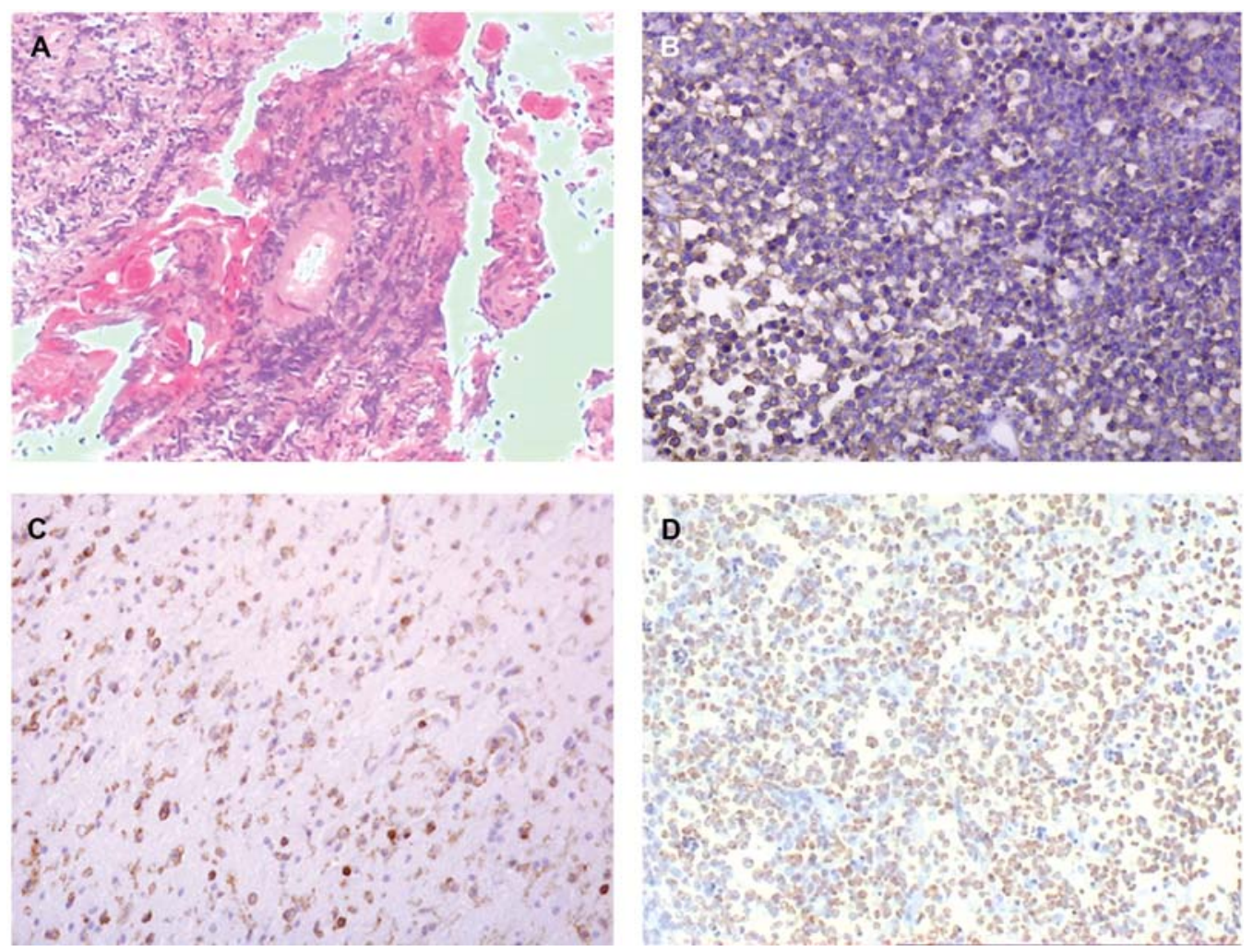

Figure 2. Histopathological findings. (A) Hematoxylin and eosin stain revealed atypical lymphoid cells clustered in an angiocentric growth pattern typical for PCNSL. (B) Immunohistochemistry using a monoclonal anti-CD20 antibody demonstrated abundant highly pleomorphic B lymphocytes. (C) Monoclonal character of lymphocyte infiltration confirmed by positive membranous immunocytochemical response against LCA (CD45). (D) The population of mitotically active cells within the tumor can be visualized by immunohistochemistry with Ki-67 antibody.

cases which included 693 cases of PCNSL. In 398 of the cases, the patients underwent craniotomies, 85 of whom had no postoperative adjuvant therapy. The median reported survival time among these 85 patients was 1 month. Only one patient survived beyond 3 years. The authors concluded that extent of resection had no effect on median survival. Tomlinson et al (73) reported their study in which among 89 cases of PCNSL, craniotomy was performed in 42 patients, and the remainder underwent biopsy. Survival was not significantly different between the groups, and the authors came to a similar conclusion that extent of resection was not associated with survival. However, interpretation of these data is difficult because no selection criteria for the craniotomy patients were provided.

Bataille et al (6) have further contended the inadvisability of open craniotomy with partial tumor resection. The 1-year survival rate in the complete resection group was $56.6 \%$, compared with $48.6 \%$ in the group underwent stereotactic biopsy alone, and $31.8 \%$ in the partial resection group. Among the three treatment variables, only partial resection achieved a statistically significant, though negative, impact on survival. The authors did not discuss the complete resection group along with whose 1-year survival rate, even though not statistically significant, is indicative of possible therapeutic benefit. Moreover, no specific information was given concerning extent of resection and patient selection criteria. In this respect most reports give information regarding survival regardless of the multiplicity of the lesion and the extent of resection. The results of surgical treatment are often based on a high percentage of multicentric lesions or partial resection (74), which makes it difficult to determine the real impact of surgery on survival.

Woodman et al (75) performed biopsy in one patient and subtotal resection in 13 patients, five of whom presenting with a solitary lesion. All patients received WBRT with a mean survival of 24.7 months. Interestingly $60 \%$ of the longterm survivors ( $>5$ years) received surgical resection of solitary lesions. Rarely but existing, Sonstein et al (76) presented a long term survivor of a surgically resected solitary PCNSL without any adjuvant RT or chemotherapy. More surprisingly, Davies et al (77) reported on a case treated with surgery without conventional therapy who survived 20 years after diagnosis.

When considering craniotomy for resection of a suspected PCNSL lesion, factors including location, size and the number of lesions present are crucial to evaluate. Bellinzona et al (78) supported the use of craniotomy in a select subset of patients who present with large, solitary, space-occupying lesions and progressive neurological deterioration. The authors described 33 cases of PCNSL in 32 patients, among which 23 patients presented with clinical signs of high intracranial pressure. The patients were treated with either gross total (15) or partial (10) removal of the tumor and stereotactic biopsy was performed in 8 cases. Nevertheless, it must be underlined that all patients who received surgery then underwent conventional therapy with RT, chemotherapy or a combination of both. The authors concluded that their data confirmed the role of RT and chemotherapy in the treatment 
of PCNSL reported previously, and that, although no benefit could be demonstrated for the role of sole surgical therapy, a selected subset of patients might benefit from craniotomy. The positive effect of surgical removal on survival could be seen among patients who received RT, chemotherapy or a combination of both, as well as all three treatment modalities had a positive and additive impact on survival (78). Of greatest significance, their data call into question the view that surgery plays a negative role in the treatment of PCNSL or that it gives no survival advantage in all cases (78).

Elder and Chen (71) argued that patients with possible pending herniation and a surgically accessible mass lesion may be considered for urgent craniotomy for debulking of the tumor and tissue diagnosis. While those without signs of herniation may undergo further preoperative evaluation, and ultimately, the majority of them will require stereotactic biopsy for tissue diagnosis.

One further point should be emphasized that though surgery as sole treatment did not appear to have improved survival over that of untreated patients, patients receiving chemotherapy and/or RT after surgery had longer survivals than those who underwent surgery alone or who received no treatment (79). However, the impact of treatment on survival has been analyzed in comparison between chemotherapy/RT combined surgery and chemotherapy/RT alone in no published reports thus far, which may partially responsible for the underestimation of surgical intervention in PCNSL.

Catheter reservoir placement. In case that intrathecal chemotherapy is required (for instance, in patients with leptomeningeal lymphoma), an Ommaya reservoir can be implanted through surgical procedure (usually during craniotomy for tumor resection). For the purpose of interstitial chemotherapy, MTX or carmustine or cytarabine can be delivered into the reservoir. The preference for intra-Ommaya administration is based on the fact that intrathecal administration is notoriously inconsistent and requires repeated intervention with lumbar puncture (80). However, patients with hydrocephalus should not receive an Ommaya reservoir.

For the purpose of brachytherapy, iridium 192 sources were delivered into the resection cavity by using a stereotactic guidance system and an image-defined, volumetrically determined target (81).

In some cases, once the diagnosis of PCNSL has been established through stereotactic biopsy, the patients could be alternatively treated with interstitial chemotherapy or/and brachytherapy without simultaneous tumor resection.

Ventriculoperitoneal shunt placement. Besides diagnostic value of stereotactic biopsy and decompression by open craniotomy resection, surgical interventions also play a role in the treatment of complications of PCNSL. PNCSL can cause generalized hydrocephalus or loculation of part of the ventricular system. Mass effect from the tumor, leptomeningeal involvement or inflammation may lead to obstruction of CSF outflow. In these cases, placement of a ventriculoperitoneal shunt is designated for diversion of CSF.

Additionally, in the presence of delayed neurotoxicity caused by combination of MTX and WBRT, treatment is limited but a handful of patients find temporary benefit from ventriculoperitoneal shunting (82). However, the use of ventriculoperitoneal shunt in PCNSL should be considered with caution since the high risk of peritoneal dissemination of tumor cells.

\section{Conclusion}

A growing number of patients have achieved high-functioning long-term survival according to current management for PCNSL. Yet population-based statistical analyses have failed to reveal improvement in survival despite the therapeutic advances (83). Chemotherapy, corticosteroids, WBRT and combined-modality therapy remain the mainstays of management for PCNSL. MTX-based, multi-agent chemotherapy currently is the treatment of choice, especially in the elderly patient population. The optimal role and timing of WBRT has yet to be established. Avoiding neurotoxicity is an important goal when deciding therapy strategy. If possible, surgical consideration is still limited to a diagnostic stereotactic biopsy to obtain tumor specimens for pathological analysis. Craniotomy for tumor resection may be indicated in patients with rapid neurological deterioration or impending herniation. Patients with clinical or neuroimaging evidence of PCNSL presenting as space occupying lesions which are amenable to resection may represent a subset of patients who merit consideration for open craniotomy to remove their tumors. Catheter reservoir placement for interstitial chemotherapy or/and brachytherapy might be taken into consideration in some cases. On account of the very nature of retrospective studies, the impact of surgery could not reach statistical significance. However, future prospective randomized study to address the role of surgery is unrealistic due to the low incidence of PCNSL and the limited number of patients eligible for surgery. Thus it is recommended that surgical procedure be used with caution and strict patient selection criteria, advisably in combination with RT and/or chemotherapy to improve the outcome.

\section{References}

1. Central Brain Tumor Registry of the United States (2005) Statistical Report: Primary brain tumors in the United States 1998-2002 (years data collected). http://www.cbtrus.org/reports// 2005-2006/2006report.pdf.

2. Abrey LE, DeAngelis LM and Yahalom J: Long-term survival in primary CNS lymphoma. J Clin Oncol 16: 859-863, 1998.

3. Olson JE, Janney CA, Rao RD, et al: The continuing increase in the incidence of primary central nervous system non-Hodgkin lymphoma: a surveillance, epidemiology, and end results analysis. Cancer 95: 1504-1510, 2002.

4. Phan TG, O'Neill BP and Kurtin PJ: Posttransplant primary CNS lymphoma. Neuro Oncol 2: 229-238, 2000.

5. Chamberlain MC: Primary CNS lymphoma. Neurology 45: 1031-1032, 1995.

6. Bataille B, Delwail V, Menet E, et al: Primary intracerebral malignant lymphoma: report of 248 cases. J Neurosurg 92: 261-266, 2000.

7. Hausdorff J, Davis E, Long G, et al: Non-Hodgkin's lymphoma of the paranasal sinuses: clinical and pathological features, and response to combined-modality therapy. Cancer J Sci Am 3: 303-311, 1997.

8. Herrlinger U, Schabet M, Bitzer M, Petersen D and Krauseneck P: Primary central nervous system lymphoma: from clinical presentation to diagnosis. J Neurooncol 43: 219-226, 1999.

9. Batchelor T and Loeffler JS: Primary CNS lymphoma. J Clin Oncol 24: 1281-1288, 2006. 
10. Plotkin SR and Batchelor TT: Primary nervous-system lymphoma. Lancet Oncol 2: 354-365, 2001.

11. Mohile NA and Abrey LE: Primary central nervous system lymphoma. Semin Raiat Oncol 17: 223-229, 2007.

12. Springer TA: Traffic signals for lymphocyte recirculation and leukocyte emigration: the multistep paradigm. Cell 76: 301-314, 1994

13. Smith JR, Braziel RM, Paoletti S, Lipp M, Uguccioni M and Rosenbaum JT: Expression of B-cell-attracting chemokine 1 (CXCL13) by malignant lymphocytes and vascular endothelium in primary central nervous system lymphoma. Blood 101: 815-821, 2003

14. Jemal A, Siegel R, Ward E, et al: Cancer statistics, 2006. CA Cancer J Clin 56: 106-130, 2006.

15. Ferreri AJ, Abrey LE, Blay JY, et al: Summary statement on primary central nervous system lymphomas from the Eighth International Conference on Malignant Lymphoma, Lugano, Switzerland, June 12-15, 2002. J Clin Oncol 21: 2407-2414, 2003.

16. Shenkier TN, Blay JY, O'Neill BP, et al: Primary CNS lymphoma of T-cell origin: a descriptive analysis from the international primary CNS lymphoma collaborative group. J Clin Oncol 23 2233-2239, 2005.

17. Tu PH, Giannini C, Judkins AR, et al: Clinicopathologic and genetic profile of intracranial marginal zone lymphoma: a primary low-grade CNS lymphoma that mimics meningioma. J Clin Oncol 23: 5718-5727, 2005.

18. Bessell EM, Hoang-Xuan K, Ferreri AJM and Reni M: Primary central nervous system lymphoma: Biological aspects and controversies in management. Eur J Cancer 43: 1141-1152, 2007.

19. Abrey LE, Batchelor TT, Ferreri AJ, et al: Report of an international workshop to standardize baseline evaluation and response criteria for primary CNS lymphoma. J Clin Oncol 23: 5034-5043, 2005

20. Pollack IF, Lunsford LD, Flickinger JC and Dameshek HL: Prognostic factors in the diagnosis and treatment of primary central nervous system lymphoma. Cancer 63: 939-947, 1989.

21. Corry J, Smith JG, Wirth A, Quong G and Liew KH: Primary central nervous system lymphoma: age and performance status are more important than treatment modality. Int J Radiat Oncol Biol Phys 41: 615-620, 1998

22. Blay JY, Lasset C, Carrie C, et al: Multivariate analysis of prognostic factors in patients with non-HIV-related primary cerebral lymphoma. A proposal for a prognostic scoring. Br J Cancer 67: 1136-1141, 1993

23. Ferreri AJ, Blay JY, Reni M, et al: Prognostic scoring system for primary CNS lymphomas: the International Extranodal Lymphoma Study Group experience. J Clin Oncol 21: 266-272, 2003.

24. Abrey LE, Ben-Porat L, Panageas KS, et al: Primary central nervous system lymphoma: the Memorial Sloan-Kettering Cancer Center prognostic model. J Clin Oncol 24: 5711-5715, 2006.

25. Ferreri AJ, Reni M, Zucca E and Cavalli F: Primary CNS lymphomas prognosis. J Clin Oncol 25: 4322-4324; author reply 4324-4325, 2007.

26. Geppert M, Ostertag CB, Seitz G and Kiessling M: Glucocorticoid therapy obscures the diagnosis of cerebral lymphoma. Acta Neuropathol 80: 629-634, 1990.

27. Weller M: Glucocorticoid treatment of primary CNS lymphoma. J Neurooncol 43: 237-239, 1999.

28. Porter AB, Giannini C, Kaufmann T, et al: Primary central nervous system lymphoma can be histologically diagnosed after previous corticosteroid use: a pilot study to determine whether corticosteroids prevent the diagnosis of primary central nervous system lymphoma. Ann Neurol 63: 662-667, 2008

29. Lai R, Rosenblum MK and DeAngelis LM: Primary CNS lymphoma: a whole-brain disease? Neurology 59: 1557-1562, 2002.

30. Shibamoto Y, Hayabuchi N, Hiratsuka J, et al: Is whole-brain irradiation necessary for primary central nervous system lymphoma? Patterns of recurrence after partial-brain irradiation. Cancer 97: 128-133, 2003

31. Blay JY, Conroy T, Chevreau C, et al: High-dose methotrexate for the treatment of primary cerebral lymphomas: analysis of survival and late neurologic toxicity in a retrospective series. J Clin Oncol 16: 864-871, 1998.
32. Nelson DF, Martz KL, Bonner H, et al: Non-Hodgkin's lymphoma of the brain: can high dose, large volume radiation therapy improve survival? Report on a prospective trial by the Radiation Therapy Oncology Group (RTOG): RTOG 8315. Int J Radiat Oncol Biol Phys 23: 9-17, 1992.

33. Bessell EM, Lopez-Guillermo A, Villa S, et al: Importance of radiotherapy in the outcome of patients with primary CNS lymphoma: an analysis of the CHOD/BVAM regimen followed by two different radiotherapy treatments. J Clin Oncol 20: 231-236, 2002

34. Gavrilovic IT, Hormigo A, Yahalom J, DeAngelis LM and Abrey LE: Long-term follow-up of high-dose methotrexatebased therapy with and without whole brain irradiation for newly diagnosed primary CNS lymphoma. J Clin Oncol 24: 4570-4574, 2006.

35. Shah GD, Yahalom J, Correa DD, et al: Combined immunochemotherapy with reduced whole-brain radiotherapy for newly diagnosed primary CNS lymphoma. J Clin Oncol 25: 4730-4735, 2007

36. Schultz C, Scott C, Sherman W, et al: Preirradiation chemotherapy with cyclophosphamide, doxorubicin, vincristine, and dexamethasone for primary CNS lymphomas: initial report of radiation therapy oncology group protocol 88-06. J Clin Oncol 14: 556-564, 1996

37. Brada M, Dearnaley D, Horwich A and Bloom HJ: Management of primary cerebral lymphoma with initial chemotherapy: preliminary results and comparison with patients treated with radiotherapy alone. Int J Radiat Oncol Biol Phys 18: 787-792, 1990.

38. Ferreri AJ, Reni M and Villa E: Therapeutic management of primary central nervous system lymphoma: lessons from prospective trials. Ann Oncol 11: 927-937, 2000.

39. Hochberg FH, Baehring JM and Hochberg EP: Primary CNS lymphoma. Nat Clin Pract Neurol 3: 24-35, 2007.

40. Ferreri AJ, Reni M, Pasini F, et al: A multicenter study of treatment of primary CNS lymphoma. Neurology 58: 1513-1520, 2002.

41. Ferreri AJ, Foppoli M, Martelli M, et al: Randomized phase II trial on primary chemotherapy (CHT) with high-dose methotrexate (MTX) alone or associated with high-dose cytarabine $(\mathrm{araC})$ for patients with primary CNS lymphoma (PSCNL). http://www.ielsg.org/documents/ielsg20lug08.pdf

42. Pels H, Schmidt-Wolf IG, Glasmacher A, et al: Primary central nervous system lymphoma: results of a pilot and phase II study of systemic and intraventricular chemotherapy with deferred radiotherapy. J Clin Oncol 21: 4489-4495, 2003.

43. Hoang-Xuan K, Taillandier L, Chinot O, et al: Chemotherapy alone as initial treatment for primary CNS lymphoma in patients older than 60 years: a multicenter phase II study (26952) of the European Organization for Research and Treatment of Cancer Brain Tumor Group. J Clin Oncol 21: 2726-2731, 2003.

44. Khan RB, Shi W, Thaler HT, DeAngelis LM and Abrey LE: Is intrathecal methotrexate necessary in the treatment of primary CNS lymphoma? J Neurooncol 58: 175-178, 2002.

45. Glass J, Gruber ML, Cher L and Hochberg FH: Preirradiation methotrexate chemotherapy of primary central nervous system lymphoma: long-term outcome. J Neurosurg 81: 188-195, 1994.

46. DeAngelis LM, Yahalom J, Thaler HT and Kher U: Combined modality therapy for primary CNS lymphoma. J Clin Oncol 10: 635-643, 1992.

47. DeAngelis LM, Seiferheld W, Schold SC, Fisher B and Schultz CJ: Combination chemotherapy and radiotherapy for primary central nervous system lymphoma: Radiation Therapy Oncology Group Study 93-10. J Clin Oncol 20: 4643-4648, 2002.

48. Poortmans PM, Kluin-Nelemans HC, Haaxma-Reiche H, et al: High-dose methotrexate-based chemotherapy followed by consolidating radiotherapy in non-AIDS-related primary central nervous system lymphoma: European Organization for Research and Treatment of Cancer Lymphoma Group Phase II Trial 20962. J Clin Oncol 21: 4483-4488, 2003.

49. Omuro AM, DeAngelis LM, Yahalom J and Abrey LE: Chemoradiotherapy for primary CNS lymphoma: an intent-to-treat analysis with complete follow-up. Neurology 64: 69-74, 2005.

50. O'Brien PC, Roos DE, Pratt G, et al: Combined-modality therapy for primary central nervous system lymphoma: long-term data from a Phase II multicenter study (Trans-Tasman Radiation Oncology Group). Int J Radiat Oncol Biol Phys 64: 408-413, 2006. 
51. Ferreri AJ, Dell'Oro S, Foppoli M, et al: MATILDE regimen followed by radiotherapy is an active strategy against primary CNS lymphomas. Neurology 66: 1435-1438, 2006.

52. Batchelor T, Carson K, O'Neill A, et al: Treatment of primary CNS lymphoma with methotrexate and deferred radiotherapy: a report of NABTT 96-07. J Clin Oncol 21: 1044-1049, 2003.

53. Herrlinger U, Kuker W, Uhl M, et al: NOA-03 trial of high-dose methotrexate in primary central nervous system lymphoma: final report. Ann Neurol 57: 843-847, 2005.

54. Mead GM, Bleehen NM, Gregor A, et al: A medical research council randomized trial in patients with primary cerebral nonHodgkin lymphoma: cerebral radiotherapy with and without cyclophosphamide, doxorubicin, vincristine, and prednisone chemotherapy. Cancer 89: 1359-1370, 2000.

55. Milpied N, Deconinck E, Gaillard F, et al: Initial treatment of aggressive lymphoma with high-dose chemotherapy and autologous stem-cell support. N Engl J Med 350: 1287-1295, 2004.

56. Abrey LE, Moskowitz CH, Mason WP, et al: Intensive methotrexate and cytarabine followed by high-dose chemotherapy with autologous stem-cell rescue in patients with newly diagnosed primary CNS lymphoma: an intent-to-treat analysis. J Clin Oncol 21: 4151-4156, 2003.

57. Cheng T, Forsyth P, Chaudhry A, et al: High-dose thiotepa, busulfan, cyclophosphamide and ASCT without whole-brain radiotherapy for poor prognosis primary CNS lymphoma. Bone Marrow Transplant 31: 679-685, 2003.

58. Montemurro M, Kiefer T, Schuler F, et al: Primary central nervous system lymphoma treated with high-dose methotrexate, high-dose busulfan/thiotepa, autologous stem-cell transplantation and response-adapted whole-brain radiotherapy: results of the multicenter Ostdeutsche Studiengruppe Hamato-Onkologie OSHO-53 phase II study. Ann Oncol 18: 665-671, 2007.

59. Illerhaus G, Marks R, Ihorst G, et al: High-dose chemotherapy with autologous stem-cell transplantation and hyperfractionated radiotherapy as first-line treatment of primary CNS lymphoma. J Clin Oncol 24: 3865-3870, 2006.

60. Colombat P, Lemevel A, Bertrand P, et al: High-dose chemotherapy with autologous stem cell transplantation as first-line therapy for primary CNS lymphoma in patients younger than 60 years: a multicenter phase II study of the GOELAMS group. Bone Marrow Transplant 38: 417-420, 2006.

61. Plotkin SR, Betensky RA, Hochberg FH, et al: Treatment of relapsed central nervous system lymphoma with high-dose methotrexate. Clin Cancer Res 10: 5643-5646, 2004.

62. Reni M, Zaja F, Mason W, et al: Temozolomide as salvage treatment in primary brain lymphomas. Br J Cancer 96: 864-867, 2007.

63. Fischer L, Thiel E, Klasen HA, et al: Prospective trial on topotecan salvage therapy in primary CNS lymphoma. Ann Oncol 17: 1141-1145, 2006.

64. Reni M, Mazza E, Foppoli M and Ferreri AJ: Primary central nervous system lymphomas: Salvage treatment after failure to high-dose methotrexate. Cancer Lett 258: 165-170, 2007.

65. Nguyen PL, Chakravarti A, Finkelstein DM, Hochberg FH, Batchelor TT and Loeffler JS: Results of whole-brain radiation as salvage of methotrexate failure for immunocompetent patients with primary CNS lymphoma. J Clin Oncol 23: 1507-1513, 2005.

66. O'Brien P, Roos D, Pratt G, et al: Phase II multicenter study of brief single-agent methotrexate followed by irradiation in primary CNS lymphoma. J Clin Oncol 18: 519-526, 2000.
67. Correa DD, DeAngelis LM, Shi W, Thaler H, Glass A and Abrey LE: Cognitive functions in survivors of primary central nervous system lymphoma. Neurology 62: 548-555, 2004.

68. Reni M and Ferreri AJ: Is withdrawal of consolidation radiotherapy an evidence-based strategy in primary central nervous system lymphomas? J Clin Oncol 22: 1165-1167; author reply 1167-1168, 2004.

69. Omuro AM, Ben-Porat LS, Panageas KS, et al: Delayed neurotoxicity in primary central nervous system lymphoma. Arch Neurol 62: 1595-1600, 2005.

70. Wefel JS, Kayl AE and Meyers CA: Neuropsychological dysfunction associated with cancer and cancer therapies: a conceptual review of an emerging target. Br J Cancer 90: 1691-1696, 2004.

71. Elder JB and Chen TC: Surgical interventions for primary central nervous system lymphoma. Neurosurg Focus 21: E13, 2006.

72. Murray K, Kun L and Cox J: Primary malignant lymphoma of the central nervous system. Results of treatment of 11 cases and review of the literature. J Neurosurg 65: 600-607, 1986.

73. Tomlinson FH, Kurtin PJ, Suman VJ, et al: Primary intracerebral malignant lymphoma: a clinicopathological study of 89 patients. J Neurosurg 82: 558-566, 1995.

74. Hayakawa T, Takakura K, Abe H, et al: Primary central nervous system lymphoma in Japan - a retrospective, co-operative study by CNS-Lymphoma Study Group in Japan. J Neurooncol 19: 197-215, 1994

75. Woodman R, Shin K and Pineo G: Primary non-Hodgkin's lymphoma of the brain. A review. Medicine (Baltimore) 64: 425-430, 1985

76. Sonstein W, Tabaddor K and Llena JF: Solitary primary CNS lymphoma: long term survival following total resection. Med Oncol 15: 61-65, 1998

77. Davies KG, Cole GC and Weeks RD: Twenty-year survival following excision of primary CNS lymphoma without radiation therapy: case report. Br J Neurosurg 8: 487-491, 1994.

78. Bellinzona M, Roser F, Ostertag H, Gaab RM and Saini M: Surgical removal of primary central nervous system lymphomas (PCNSL) presenting as space occupying lesions: a series of 33 cases. Eur J Surg Oncol 31: 100-105, 2005.

79. Reni M, Ferreri AJM, Garancini MP and Villa E: Therapeutic management of primary central nervous system lymphoma in immunocompetent patients: Results of a critical review of the literature. Ann Oncol 8: 227-234, 1997.

80. Shapiro W, Young D and Mehta B: Methotrexate: Distribution in cerebrospinal fluid after intravenous, ventricular and lumbar injections. N Engl J Med 293: 161-166, 1975.

81. Apuzzo ML, Petrovich Z, Luxton G, Jepson JH, Cohen D and Breeze RE: Interstitial radiobrachytherapy of malignant cerebral neoplasms: rationale, methodology, prospects. Neurol Res 9: 91-100, 1987.

82. Thiessen B and DeAngelis LM: Hydrocephalus in radiation leukoencephalopathy: results of ventriculoperitoneal shunting. Arch Neurol 55: 705-710, 1998

83. Panageas KS, Elkin EB, DeAngelis LM, Ben-Porat L and Abrey LE: Trends in survival from primary central nervous system lymphoma, 1975-1999: A population-based analysis. Cancer 104: 2466-2472, 2005. 